\title{
Berberry Extract Reduces Neuronal Damage and $N$-Methyl-D-aspartate Receptor 1 Immunoreactivity in the Gerbil Hippocampus after Transient Forebrain Ischemia
}

\author{
Ki-Yeon Yoo, ${ }^{a}$ In Koo Hwang, ${ }^{a, b}$ Beong Ou Lim,,${ }^{c}$ Tae-Cheon Kang, ${ }^{a}$ Dong-Woo Kim, ${ }^{b}$ \\ Sang Moo KIm, ${ }^{d}$ Hyeon Yong LeE, ${ }^{e}$ Jong Dai KIm, ${ }^{e}$ and Moo Ho Won ${ }^{*, a}$ \\ ${ }^{a}$ Department of Anatomy, College of Medicine, Hallym University; Chunchon 200-702, South Korea: ${ }^{b}$ Natural F\&P Co., \\ LTD; Chunchon 200-163, South Korea: ${ }^{c}$ Department of Applied Biochemistry, Konkuk University; Chungju, Chungbuk, \\ 380-701, South Korea: ' Faculty of Marine Bioscience and Technology, Kangnung National University; Gangneung \\ 210-702, South Korea: and ${ }^{e}$ School of Biotechnology and Bioengineering, Kangwon National University; Chunchon \\ 200-701, South Korea. Received August 2, 2005; accepted December 27, 2005
}

In the present study, we studied the neuroprotective effects of berberry extract (BE) against ischemic damage and the temporal and spatial alterations of $N$-methyl-D-aspartate receptor type 1 (NR1) and NR2A/2B immunoreactivities in the gerbil hippocampal CA1 region after transient ischemia to examine anti-ischemic effects and its role in transient forebrain ischemia. In the vehicle-treated group, the percentage of cresyl violet positive pyramidal cells in the CA1 region was about $11.4 \%$ compared to the sham-operated group $4 \mathrm{~d}$ after ischemic insult. BE showed neuroprotective effects against ischemic damage after ischemia-reperfusion. In the BEtreated groups, about $60-75 \%$ of CA1 pyramidal cells were stained with cresyl violet $4 \mathrm{~d}$ after ischemic insult. We observed the percentage of berberine $(7.45+0.85 \mathrm{mg} / \mathrm{g}$ in BE) by HPLC, which is active ingredient of BE. NR1 immunoreactivity in the stratum pyramidale of the CA1 region in the vehicle-treated group was significantly increased at $30 \mathrm{~min}$ after transient forebrain ischemia, while at this time the NR1 immunoreactivity in the BE-treated groups was significantly low compared to the vehicle-treated group. The pattern of NR2A/B immunoreactivity in the stratum pyramidale of the BE-treated group and its protein levels were similar to that in the vehicle-treated group after ischemic insult. These results suggest that $B E$ has potent neuroprotective effects against ischemic damage via the reduction of NR1 activity.

Key words transient forebrain ischemia; hippocampal CA1 region; neuroprotection; berberry extract; $N$-methyl-D-aspartate receptor; gerbil

Transient forebrain ischemia, produced by prolonged deprivation of blood flow to the brain, results in the insidious delayed degeneration of specific vulnerable neurons within the

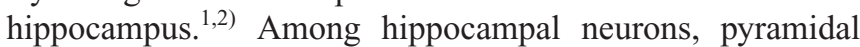
cells of the hippocampal CA1 region are most vulnerable to cerebral ischemia. ${ }^{1,2)}$ Death of the CA1 pyramidal cells following cerebral ischemia causes a variety of neurological dysfunction such as depression and memory deficits. ${ }^{3,4)}$

In traditional Eastern medicine, the extracts of various Berberidaceae (Berberis aquifolium, Berberis vulgaris and Berberis aristata) are used for rheumatic complaints and other types of chronic inflammation. ${ }^{5,6}$ It has been reported that the functional components of the extracts were alkaloid components such as berberine, oxyacanthine, berbamine and palmatine. ${ }^{5)}$ The anti-inflammatory and immunosuppressive activity of the Berberidaceae extract is well established. ${ }^{7,8)}$ Moreover, cardiotonic ${ }^{9)}$ and antiarrhythmic ${ }^{10,11)}$ activities have also been reported. Despite the considerable works done on berberic species in inflammation, cardiotonic and antiarrhythmic effects, its effects and roles in ischemic damage in the brain remains to be elucidated.

It has been postulated that the neuronal hyperactivity due to an elevation of extracellular excitatory amino acids during ischemia leads to a massive increase in intracellular-free $\mathrm{Ca}^{2+}$, which may trigger the process of neuronal degeneration. ${ }^{12-14)}$ In the present study, therefore, we investigated the percentage of berberine by HPLC, which is active ingredient, and the neuroprotective effects of crude berberry extract (BE) against ischemic damage and the temporal and spatial alterations of $\mathrm{N}$-methyl-D-aspartate receptor type 1 (NR1) and NR2A/B, the major excitatory amino acid receptors, immunoreactivities in the hippocampus induced by transient ischemia to examine anti-ischemic effects and their roles after transient forebrain ischemia in gerbils.

\section{MATERIALS AND METHODS}

Experimental Animals Male Mongolian gerbils (Meriones unguiculatus) obtained from the Experimental Animal Center, Hallym University, Chunchon, South Korea were used in this study. The animals were housed at constant temperature $\left(23^{\circ} \mathrm{C}\right)$ at $60 \%$ relative humidity under a fixed $12 \mathrm{~h}$ light/dark cycle and had free access to food and water. Procedures involving animals and their care conformed with the our institution's guidelines, which are in compliance with current international laws and policies (NIH Guide for the Care and Use of Laboratory Animals, NIH Publication No. 85-23, 1985, revised 1996). All experiments were conducted to minimize the number of animals used and suffering caused.

Plant Materials and Extraction Korean berberry (Berberis koreana) was collected in the area around Gyeonggi-do (South Korea) in September 2001. The plants were authenticated and the voucher specimen was deposited in the departmental herbarium of Kangwon National University. For the preparation of the ethanol extract of Berberis koreana, whole plants of theBerberis koreana were dried and ground into fine powder. The powder $(100 \mathrm{~g})$ was dispersed in $99.8 \%$ ethanol, and refluxed for $1 \mathrm{~h}$ at $70^{\circ} \mathrm{C}$. This extraction procedure was repeated three times. The ethanol extract 
was evaporated and dried under vacuum to get residue $(9.8 \mathrm{~g})$. One hundred micrograms of ethanol extract of Berberis koreana (BE) was suspended in $10 \mathrm{ml}$ corn oil.

Quantification of Berberine in BE by HPLC: Chemicals and Reagents Structure of berberine is shown in the Fig. 1. Berberine was purchased from Sigma-Aldrich (St. Louis, MO, U.S.A.). Acetonitrile and methanol (HPLC grade) were purchased from Labscan (Dublin, Ireland). Phosphoric acid was of analytical-reagent grade. Ultra-pure distilled water with a resistivity greater than $18 \mathrm{M} \Omega$ was used.

Quantification of Berberine BE by HPLC: Instruments and Liquid Chromatography The HPLC system (Dionex, Sunnyvale U.S.A.) consisted of a Dionex Model P580 multisolvent delivery system equipped with an ASI-100 automated sample injector and a Model STH 585 column oven and a Model UVD170S detector. Peak areas were calculated with a Chromeleon integrator (Version 6.30). The mobile phase was composed of $0.03 \%(\mathrm{v} / \mathrm{v})$ phosphoric acid-acetonitrile with gradient elution ( $0 \mathrm{~min}, 90: 10 ; 45 \mathrm{~min}, 0: 100$; 45-55 min, $0: 100 ; 55-60 \mathrm{~min}, 90: 10 ; 70 \mathrm{~min}, 90: 10$ ). The solvents were filtered through a $0.45 \mu \mathrm{m}$ Millipore filter and degassed prior to using. A Luna C18 reversed-phase column (Phenomenex, $250 \times 4.6 \mathrm{~mm}$ I.D.) and a guard column (ODS, Phenomenex, $4 \mathrm{~mm} \times 3.0 \mathrm{~mm}$ I.D.) were used. The flow-rate was $0.8 \mathrm{ml} / \mathrm{min}$ with UV absorbance detection at $280 \mathrm{~nm}$. The operating temperature was maintained at $30^{\circ} \mathrm{C}$.

Quantification of Berberine in BE by HPLC: Preparation of Standard and BE Solution Berberine was accurately weighed and dissolved in $70 \%$ methanol to give serial concentrations in the ranges $0.010-1.00 \mu \mathrm{g} / \mathrm{ml}$. Calibration graphs were plotted by linear regression of the peak-area ratio with concentration. The concentration of $\mathrm{BE}$ for quantification was used $2.0 \mathrm{mg} / \mathrm{ml}$ with $70 \%$ methanol. All samples were filtered through a $0.45 \mu \mathrm{m}$ Millipore filter and injected for HPLC analysis to calculate the quantity.

Induction of Ischemia Gerbils weighing 65-75 g were placed under general anesthesia with a mixture of $2.5 \%$ isoflurane (Baxtor, U.S.A.) in 33\% oxygen and $67 \%$ nitrous oxide. A midline ventral incision was made in the neck. Both common carotid arteries were isolated, freed of nerve fibers, and occluded them with non-traumatic aneurysm clips. Complete interruption of blood flow was confirmed by observing the central artery in the eyeball using an ophthalmoscope. After 5 min occlusion, the aneurysm clips were removed from the common carotid arteries. Restoration of blood flow (reperfusion) was directly observed under the ophthalmoscope. We maintained the body (rectal) temperature under free-regulating or normothermic $\left(37 \pm 0.5^{\circ} \mathrm{C}\right)$ conditions with a rectal temperature probe (TR-100; YSI, U.S.A.) and thermometric blanket before, during the surgery and after the surgery until the animals fully recovered from anesthesia. Sham-operated animals served as controls: these sham-operated animals were subjected to the same surgical procedures except that the common carotid arteries were not occluded. ${ }^{15-17)}$

Treatment of BE The BE was injected orally using the jonde $(60 \mathrm{mg} / \mathrm{kg})$ at $30 \mathrm{~min}$ before or after the occlusion of both common carotid arteries. Animal groups were 4 groups: 1) sham-operated group, 2) vehicle-treated group, 3) BE-pretreated group and 4) BE-post-treated group.

Tissue Preparation To obtain the exact data in this study, tissues of sham-operated and operated animals were simultaneously processed at the same moment in the same day. For histology, the animals were anesthetized with pentobarbital sodium and perfused transcardially with $0.1 \mathrm{M}$ phosphate-buffered saline (PBS, $\mathrm{pH}$ 7.4) followed by $4 \%$ paraformaldehyde in $0.1 \mathrm{M} \mathrm{PB}(\mathrm{pH} \mathrm{7.4)}$ at the designated times after the surgery. Brains were removed and postfixed in the same fixative for $6 \mathrm{~h}$. The brain tissues were cryoprotected by infiltration with $30 \%$ sucrose overnight. Thereafter the tissues were frozen and serially sectioned in $30 \mu \mathrm{m}$ thick coronal sections on a cryostat and the sections were collected in six-well plates containing PBS.

Cresyl Violet Staining To elucidate the protective effects of BE on ischemic damage, we examined cresyl violet positive cells in the hippocampal CA1 region using a commonly used protocol for cresyl violet staining ${ }^{17)}$ after BE treatment. Four days after ischemic insult, 7 animals in the 4 groups were used for Analysis of BE effects on ischemic damage.

Immunohistochemistry for NR1 and NR2A/B Freefloating sections of the sham-operated $(n=3$ at each time point) and operated animals ( $n=7$ at each time point) at designated times $(30 \mathrm{~min}, 3 \mathrm{~h}, 6 \mathrm{~h}, 12 \mathrm{~h}$ and $12 \mathrm{~d}$ after the surgery) were simultaneously processed by immunohistochemistry at the same moment in the same day. The sections were treated with pepsin (DAKO, Carpinteria, CA, U.S.A.). Before pepsin pretreatment, $0.2 \mathrm{~N} \mathrm{HCl}$ was warmed up to $37^{\circ} \mathrm{C}$ in a water bath. Frozen aliquots containing $100 \mathrm{mg} / \mathrm{ml}$ of pepsin solution were thawed just before use, and added to $0.2 \mathrm{~N} \mathrm{HCl}$. Then, the sections pre-warmed at $37^{\circ} \mathrm{C}$ in distilled water were transferred to the pepsin/ $\mathrm{HCl}$ solution. After a given incubation time, the sections were briefly washed with phosphate-buffered saline (PBS, $\mathrm{pH}$ 7.2), and processed for immunohistochemistry. ${ }^{18)}$ The sections were incubated with rabbit anti-NR1 antiserum $(1: 1000$, Chemicon, U.S.A.) or rabbit anti-NR2A/B antiserum (1:1000, Chemicon, U.S.A.) in PBS containing $0.3 \%$ Triton X-100 and $2 \%$ normal goat serum overnight at room temperature. After washing 3 times for $10 \mathrm{~min}$ with $\mathrm{PBS}$, they were incubated sequentially, in goat anti-rabbit IgG and Vectastain (Vector, U.S.A.), diluted $1: 200$ in the same solution as the primary antiserum. Between incubations, the tissues were washed with PBS 3 times for $10 \mathrm{~min}$ each. The sections were visualized using 3,3'-diaminobenzidine tetrachloride (DAB, Fluka, U.S.A.) in $0.1 \mathrm{~m}$ Tris buffer and mounted on gelatin-coated slides. Immunoreactions were observed under an Axioscope microscope (Carl Zeiss, Germany).

In order to establish the specificity of the immunostaining, a negative control test was carried out by substituting normal rabbit serum for primary antibody or by omitting the primary antibody. The negative control showed the absence of immunoreactivity in any structures.

Western Blot Analysis To confirm the changes of NR1 and NR2A/B levels in the hippocampal CA1 region in vehicle- and BE-treated groups after transient forebrain ischemia, at designated times ( $30 \mathrm{~min}, 3 \mathrm{~h}$ and $12 \mathrm{~h}$ after the surgery), vehicle-treated ( $n=5$ at each time point) and BE-treated animals ( $n=5$ at each time point) were sacrificed and used for the Western blot study. After sacrificing them and removing the hippocampus, the tissues were sectioned in $400 \mu \mathrm{m}$ thick coronal sections on a Vibratome (Leica, Germany) and hip- 
pocampal CA1 region was dissected with surgical blade. The tissues were homogenized in $50 \mathrm{~mm}$ PBS ( $\mathrm{pH} 7.4$ ) containing EGTA (pH 8.0), 0.2\% NP-40, 10 mм EDTA (pH 8.0), 15 mм sodium pyrophosphate, $100 \mathrm{~mm} \beta$-glycerophosphate, $50 \mathrm{~mm}$ $\mathrm{NaF}, 150 \mathrm{~mm} \mathrm{NaCl}, 2 \mathrm{~mm}$ sodium orthvanadate, $1 \mathrm{~mm}$ PMSF and $1 \mathrm{~mm}$ DTT. After centrifugation, the protein concentration was determined in the supernatants using a Micro BCA protein assay kit with bovine serum albumin as a standard (Pierce Chemical, U.S.A.). Aliquots containing $20 \mu \mathrm{g}$ of total protein were boiled in loading buffer containing $150 \mathrm{~mm}$ Tris (pH 6.8), 3 mм DTT, 6\% SDS, 0.3\% bromophenol blue and $30 \%$ glycerol. Then, each aliquot was loaded onto a $10 \%$ polyacryamide gel. After electrophoresis, the gels were transferred to nitrocellulose transfer membranes (Schleicher and Schuell, U.S.A.). To reduce background staining, the filters were incubated with 5\% non-fat dry milk in PBS containing $0.1 \%$ Tween 20 for 45 min followed by incubation with rabbit anti-NR1 antiserum $(1: 2000)$ and rabbit anti-NR2A/B antiserum $(1: 2000)$, with peroxidase conjugated horse anti-rabbit IgG (Sigma, U.S.A.), and then with ECL kit (Amersham, U.S.A.).

Quantification of Data and Statistical Analysis The number of survived pyramidal cells in the stratum pyramidale within the CA1 region was counted using an Axiophot light microscope (Carl Zeiss, Germnay) photomicroscope at a magnification of $400 \times$. Histologic analysis was performed by a blinded observer and the average of the right and left survived cell numbers (neurons per $1 \mathrm{~mm}$ liner length) in a single section of dorsal hippocampus was calculated as reported by Kirino group. ${ }^{18)}$ Five sections of cresyl violet staining from each animal were used for counting.

For quantitative analysis of NR1 or NR2A/B immunoreactivities in the hippocampal CA1 region, 15 sections per animal were taken from corresponding levels of the CA1 region. Tissue images taken from sections were obtained through an Axiophot light microscope (Carl Zeiss, Germnay) connected via $\mathrm{CCD}$ camera to a $\mathrm{PC}$ monitor. At a magnification of 25 $50 \times$, the regions were outlined on the monitor and their areas were measured. Each studied field in each tissue was selected within the CA1 region containing all layers. NR1 or NR2A/B immunoreactivities were captured with an Applescanner, and the brightness and contrast of each image file was uniformly calibrated using Adobe Photoshop version 2.4.1, followed by analysis using NIH Image 1.59 software. The intensity of NR1 or NR2A/B immunoreactivities was evaluated by means of a relative optical density (ROD) value which value was obtained after the transformation of mean gray values into ROD using the formula.

Inter-animal differences in each group, as well as interexperimental differences, were not statistically significant. Shown values represent the means of experiments performed for each hippocampal area. Shown values represent the means of experiments performed for each marker and each area. Differences among the means were statistically analyzed by one-way analysis of variance followed by the Duncan's new multiple range method or Newman-Keuls test to elucidate the ischemia-related changes of NR1 and NR2A/B or to investigate the protective effects of $\mathrm{BE}$ compared to that of vehicle-treated group. $p<0.05$ was considered significant.

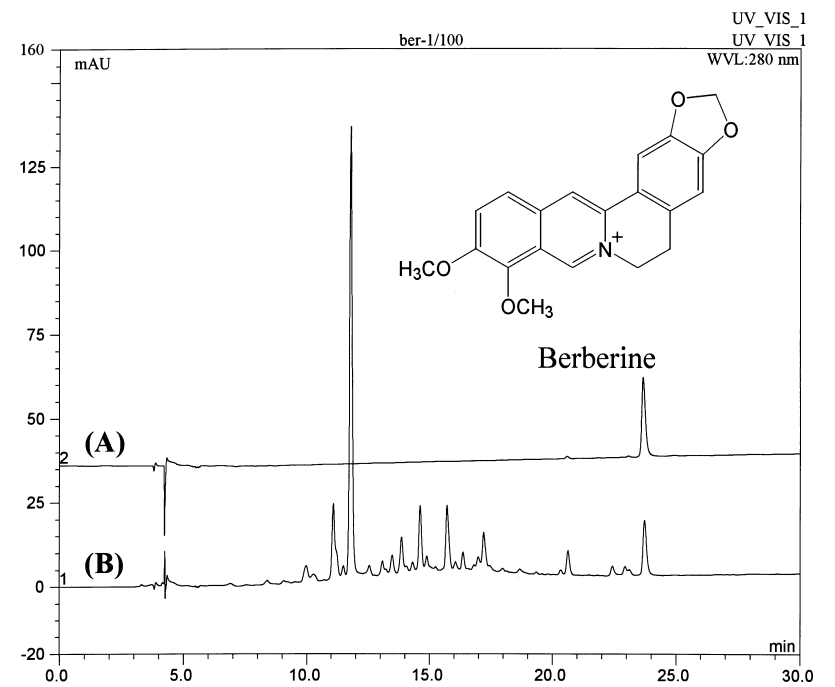

Fig. 1. Typical HPLC Chromatograms at $280 \mathrm{~nm}$ of Berberine (A) and Korean Berberry (B) Extracted Solution

The mobile phase was composed of $0.03 \%(\mathrm{v} / \mathrm{v})$ phosphoric acid-acetonitrile with gradient elution $(0 \mathrm{~min}, 90: 10 ; 45 \mathrm{~min}, 0: 100 ; 45-55 \mathrm{~min}, 0: 100 ; 55-60 \mathrm{~min}$, $90: 10 ; 70 \mathrm{~min}, 90: 10$ ). A Luna C18 reversed-phase column (Phenomenex, $250 \times 4.6$ $\mathrm{mm}$ I.D.) and a guard column (ODS, Phenomenex, $4 \mathrm{~mm} \times 3.0 \mathrm{~mm} \mathrm{I.D.)}$ were used. The flow-rate was $0.8 \mathrm{ml} / \mathrm{min}$ with UV absorbance detection at $280 \mathrm{~nm}$.

\section{RESULTS}

Quantification of Berberine in BE The HPLC method for the determination of berberine was developed based on known methods ${ }^{19)}$ and optimized (Fig. 1). According to this method, the BE contained a mean content of berberine of $7.45 \pm 0.85 \mathrm{mg} / \mathrm{g}$. The linear regression of berberine showed very good linear relationships between peak-area ratio and concentration $(R=1)$.

Neuroprotective Wffects of BE against Ischemic Damage The neuroprotective effects of BE on pyramidal cells in the hippocampal CA1 region was evaluated by measuring the neuronal cell density $4 \mathrm{~d}$ after ischemia/reperfusion (Figs. 2, $3)$. In the vehicle-treated group, the percentage of cresyl violet positive CA1 pyramidal cells in the stratum pyramidale was about $11.4 \%$ compared to the sham-operated group due to delayed neuronal death (Figs. 2A-D, 3). CA1 pyramidal cell density in the BE-treated ischemic group was significantly increased compared to the vehicle-treated group $4 \mathrm{~d}$ after transient forebrain ischemia (Figs. 2E-H, 3). In the BE-pre-treated group, about $75 \%$ of CA1 pyramidal cells compared to the sham-operated group were stained with cresyl violet $4 \mathrm{~d}$ after ischemic insult (Fig. 3). In the BE-posttreated group, about $60 \%$ of CA1 pyramidal cells compared to the sham-operated group were detected (Fig. 3).

Changes in NR1 Immunoreactivity In the present study, we found that NR1 immunoreactivity in the BE-treated group was lower than that in the vehicle-treated group in the hippocampal CA1 region after ischemic insult in gerbils (Figs. 4, 5A). Weak NR1 immunoreactivity was detected mainly in the stratum pyramidale of the CA1 region in the vehicle-treated sham-operated group (Fig. 4A). NR1 immunoreactivity in the stratum pyramidale of the CA1 region in the BE-treated sham-operated group was lower than that of the vehicle-treated sham-operated group (Figs. 4E, 5A). At 30 min after ischemic insult, NR1 immunoreactivity in the 

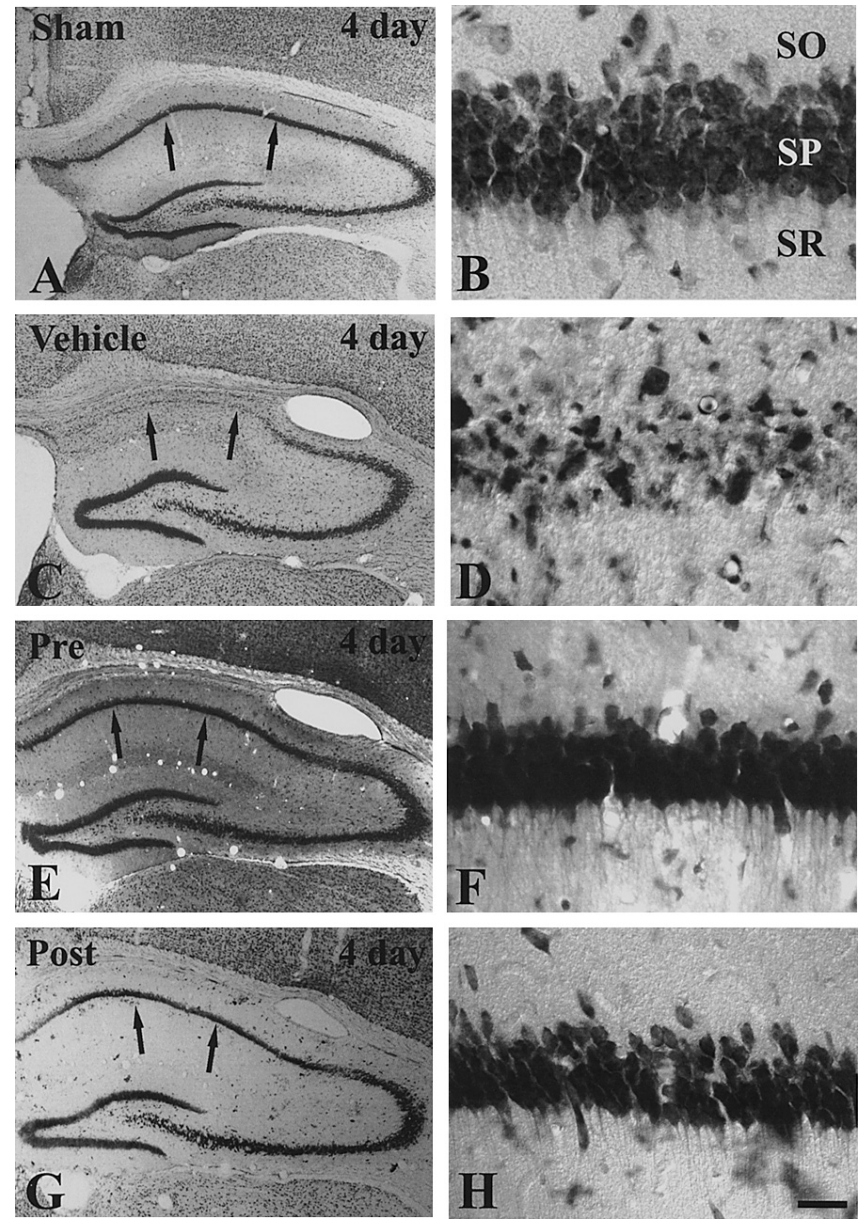

Fig. 2. Photographs of the Hippocampus Stained with Cresyl Violet in Sham-Operated Group (A, B), Vehicle-Treated Group (C, D), BE-PreTreated Group (E, F) and BE-Post-Treated Group $(\mathrm{G}, \mathrm{H}) 4 \mathrm{~d}$ after Ischemic insult

In the vehicle-treated group (C), the stratum pyramidale (SP, arrows) of the CA1 region is poorly stained with cresyl violet, a few cells (D) in the SP are stained with cresyl violet. In the BE-treated groups $(\mathrm{E}-\mathrm{H})$, many pyramidal cells are stained with cresyl violet in the SP. SO, stratum oriens; SR, stratum radiatum. Bar $=800 \mu \mathrm{m}$ (A, C, E, G), $50 \mu \mathrm{m}(\mathrm{B}, \mathrm{D}, \mathrm{F}, \mathrm{H})$.

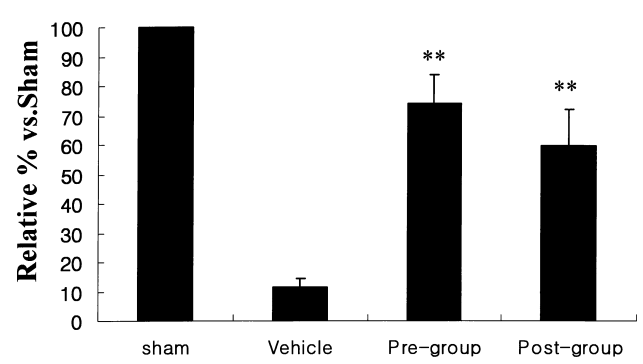

Fig. 3. Relative Numberic Analysis of Cresyl Violet Positive CA1 Pyramidal Cells in Vehicle-Treated and BE-Treated Groups versus Sham-Operated Group after Ischemic Insult

Significantly different from the vehicle treated group, $* * p<0.01$. The bars indicate the means \pm S.D.

vehicle-treated group was significantly increased in the stratum pyramidale (Figs. 4B, 5A). At this time after ischemia, NR1 immunoreactivity in the BE-treated group was increased in the stratum pyramidale, but the NR1 immunoreactivity was significantly low compared to that of the vehicletreated group (Figs. 4F, 5A). From $3 \mathrm{~h}$ after ischemic insult,
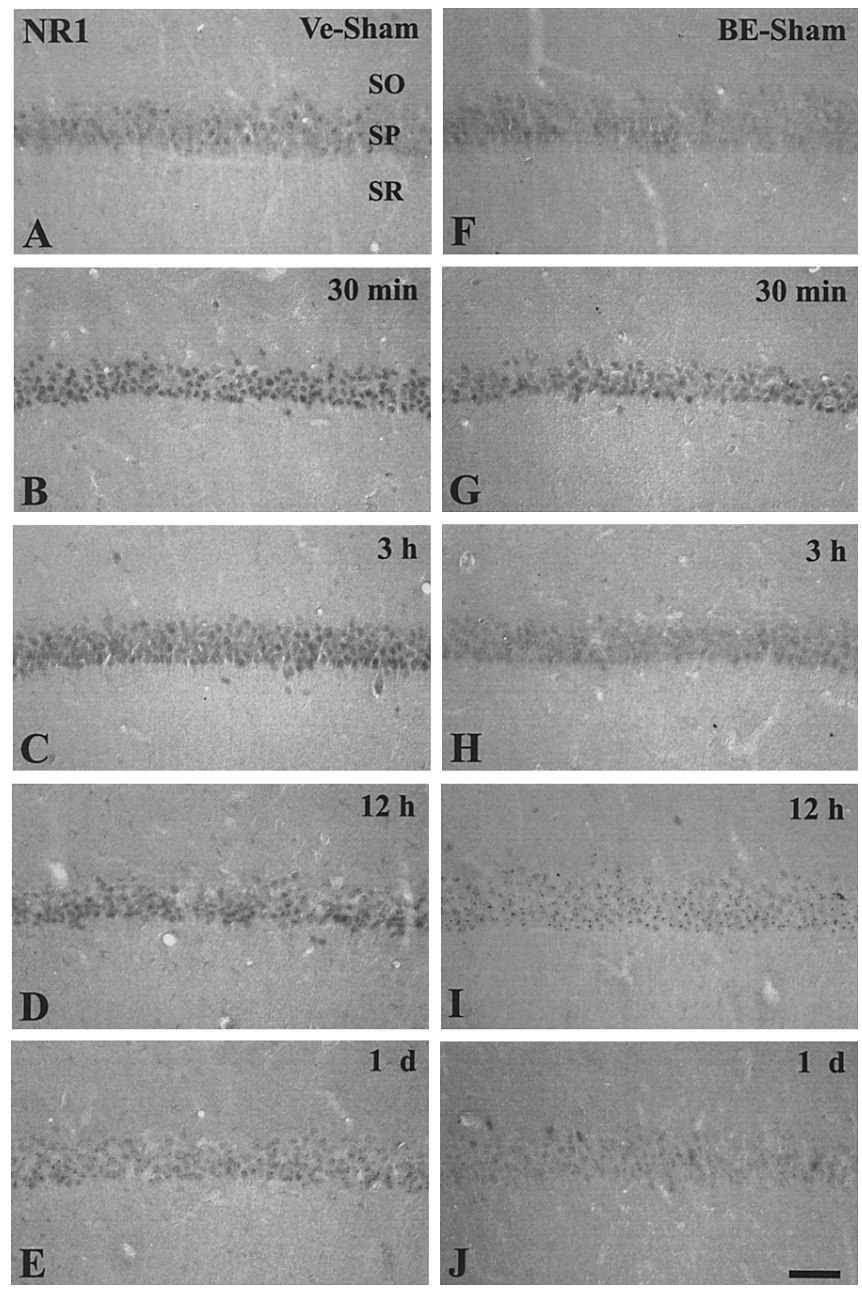

Fig. 4. Immunohistochemical Staining for NR1 in the Hippocampal CA1 Region in Vehicle-Treated Groups (A-E) and in BE-Treated Groups ( $\mathrm{F}-\mathrm{J}$ )

NR1 immunoreactivity is detected in the stratum pyramidale (SP) of the sham-operated group after vehicle treatment (A) and BE-treated sham-operated group (F). At $30 \mathrm{~min}$ after ischemic insult, NR1 immunoreactivity is elevated in the SP in both groups (B, G); NR1 immunoreactivity in the BE-treated group is lower than that in the vehicle-treated group. From $3 \mathrm{~h}$ after ischemic insult, NR1 immunoreactivity is decreased time-dependently in both groups $(\mathrm{C}-\mathrm{E}, \mathrm{H}-\mathrm{J})$. Note that NR1 immunoreactivity in the BE-treated group is lower than that in the vehicle-treated group. SO, stratum oriens; SR, stratum radiatum. Bar $=100 \mu \mathrm{m}$.

NR1 immunoreactivity became to decrease time-dependently in the CA1 region in both groups, and was low at $24 \mathrm{~h}$ after ischemia/reperfusion in both groups (Figs. $4 \mathrm{C}-4 \mathrm{I}, 4 \mathrm{G}-4 \mathrm{~J}$, $5 \mathrm{~A})$.

Changes in NR2A/B Immunoreactivity In the present study, the change of NR2A/B immunoreactivity in the BEtreated group was similar to that in the vehicle-treated group in the CA1 region after ischemia/reperfusion (Fig. 5B). NR2A/B immunoreactivity in the sham-operated group after $\mathrm{BE}$ treatment was similar to that in the vehicle-treated shamoperated group (Fig. 5B). At $3 \mathrm{~h}$ after ischemic insult, NR2A/B immunoreactivity in the stratum pyramidale was highest in both groups after ischemia (Fig. 5B). Thereafter NR2A/B immunoreactivity was decreased time-dependently after ischemia in both groups (Fig. 5B).

Changes in NR1 and NR2A/B Protein Levels In the present study, we found that NR1 protein level in the BEtreated group was lower than that in the vehicle-treated group in the hippocampal CA1 region after ischemic insult in ger- 


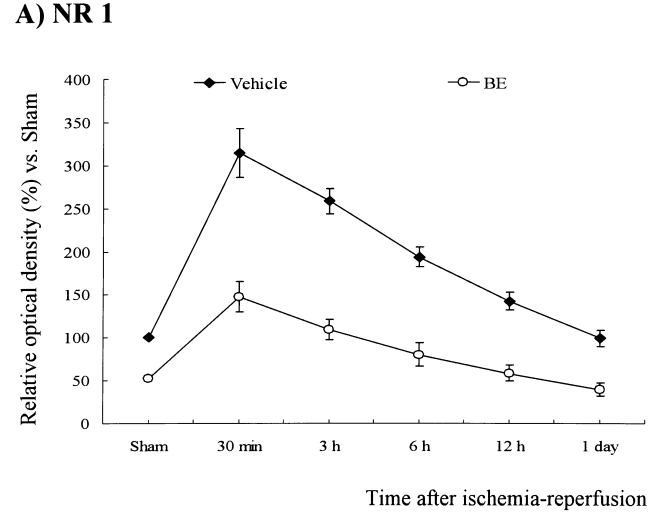

B) NR 2A/B

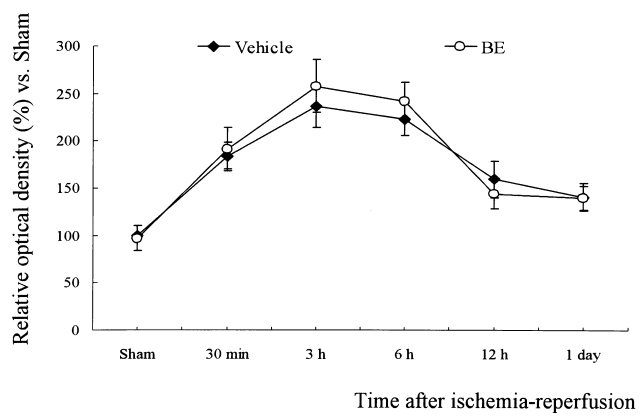

Fig. 5. The ROD of NR1 (A) and NR2A/B (B) Immunoreactivity in the Gerbil Hippocampal CA1 Region after Ischemic Insult in Vehicle-Treated Groups and in BE-Treated Groups

${ }^{\mathrm{a}} p<0.05$, significant differences from sham-operated group, ${ }^{\mathrm{b}} p<0.05$, significant differences from vehicle- and BE-treated groups. The bars indicate the means \pm S.D.

bils (Fig. 6). At $30 \mathrm{~min}$ after ischemic insult, NR1 protein level was significantly increased in the vehicle-treated group; while NR1 protein level in the BE-treated group was significantly low compared to that of the vehicle-treated group (Fig. 6). Thereafter, protein levels of NR1 in both groups were significantly decreased time-dependently after ischemic insult (Fig. 6). However, the protein levels of NR2A/B in BE-treated group were similar to that of vehicle-treated group (Fig. 6). At $3 \mathrm{~h}$ after ischemia-reperfusion, NR2A/B protein levels were significantly increased in both vehicle and BE-treated group, and thereafter their protein levels were decreased in both groups. The difference of NR2A/B protein levels in vehicle and BE-treated groups was not detected (Fig. 6)

\section{DISCUSSION}

Cell death in stroke partially results from the accumulation of high local concentration of excitatory amino acids, in particular, glutamate and enormous increase of reactive oxygen species. ${ }^{14,20-22)}$ High concentrations of glutamate over-activate the NR to allow calcium to enter neurons, thereby leading to the activation of calcium-dependent enzymes and cell death. ${ }^{23,24)}$

In the present study, we observed that BE has protective effects against ischemic damage after ischemia/reperfusion. In the BE-treated groups, about $60-75 \%$ of hippocampal CA1 pyramidal cells were stained with cresyl violet $4 \mathrm{~d}$ after ischemic insult. To confirm the role of $\mathrm{BE}$ on neuroprotective

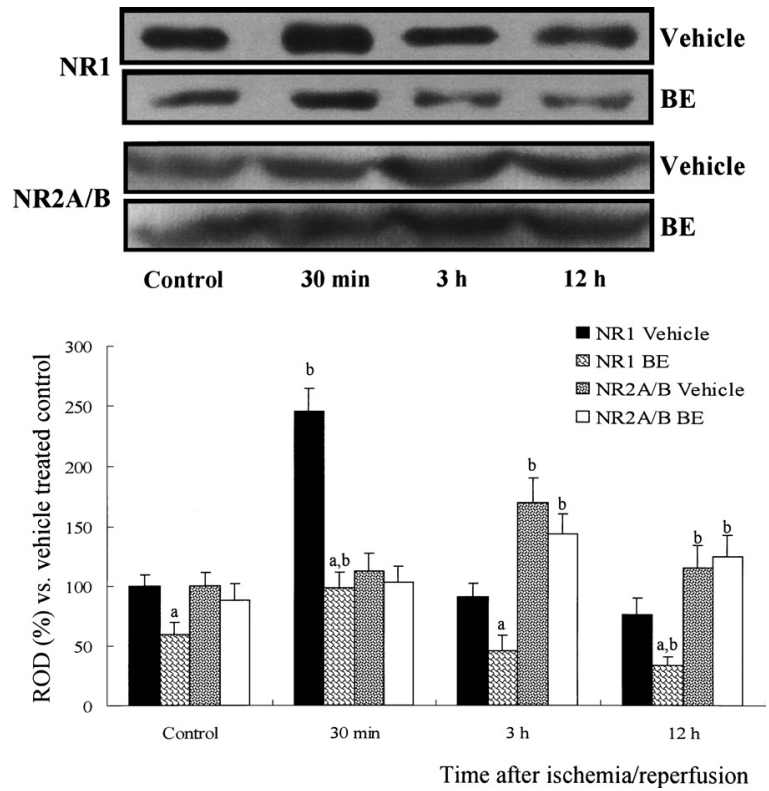

Fig. 6. The Western Blot Analyses of NR1 and NR2A/B in the Gerbil Hippocampal CA1 Region after Ischemic Insult in Vehicle-Treated and BETreated Groups

The ROD of immunoblot band is also represented. ${ }^{a} p<0.05$, significant differences from vehicle treated group, ${ }^{\mathrm{b}} p<0.05$, significant differences from control groups. The bars indicate the means \pm S.D.

effects against ischemic damage, we investigated the chronological changes of NR1 and NR2A/B immunoreactivity in the hippocampal CA1 region early time after ischemia/reperfusion. NR1 immunoreactivity was significantly increased at 30 min after ischemia in the vehicle-treated group, while in the BE-treated group, NR1 immunoreactivity was slightly increased at this time point. NR2A/B immunoreactivity and protein levels were increased at $3 \mathrm{~h}$ after ischemic insult in both vehicle- and BE-treated groups, and its immunoreactivity and protein level were slightly decreased time-dependently after ischemia in both groups.

This result suggests that a compensatory reaction, protecting against the excitotoxicity occurs for a reduction of NR responsiveness. NR is inactivated by intracellular $\left.\mathrm{Ca}^{2+},{ }^{25}\right)$ and this inactivation is regulated by $\mathrm{C}$-terminal splicing of the NR1 subunit. ${ }^{26}$ ) Thus the diminution of NR1 immunoreactivity may result from alternative splicing of the C-terminal of NR1, because the NR1 antibody used in this experiment was developed with synthetic a C-terminal to detect NR1-1 and NR1-2, but not NR1-3 or NR1-4. ${ }^{27)}$ Furthermore, the increased NR2A/B immunoreactivity and protein level may be closely related to the alternative splicing of the NR1C-terminal for the regulation of NR-mediated neuronal excitability, because the NR1 splice form requires NR2 molecules for the efficient expression. ${ }^{28)}$ Hence, the reduction of NR1 immunoreactivity and the increase of NR2A/B immunoreactivity and protein level in the ischemic hippocampal CA1 region at early time after $\mathrm{BE}$ treatment may decrease the glutamate-mediated intracellular calcium levels.

In a previous study, NR1 immunoreactivity was detected at the highest level in the strata oriens and radiatum of the CA1 region, and high levels were also seen in most other neuropil layers of the CA1 and CA3 regions and of the dentate gyrus in mouse hippocampus. ${ }^{29)}$ In the present study, we observed 
that NR1 immunoreactivity was mainly detected in the stratum pyramidale, not strata radiatum or oriens of sham-operated animals.

Berberine is isoquinoline alkaloid with a long history of medicinal used in both Ayurvedic and Chinese medicine. In recent studies, berberine has been shown to have multiple pharmacological actions including anticerebral ischemic effect, inhibition of thromboxane A2 synthesis $^{30)}$ and a partial agonist of platelet alpha 2 adenoceptor. ${ }^{31)}$

It has been reported that berberine could block the delayed rectifier potassium current, inward rectifier potassium current and L-type calcium current in guinea pig ventricular myocytes $^{32)}$ and in rat CA1 hippocampal neurons. ${ }^{33)}$ These results support our study that berberine or BE has potent inhibitory effects against calcium influx via NR1 on hippocampal CA1 pyramidal cells. Berberine is also known to possess antipyretic effects using Freund's complete adjuvant-induced subcutaneous pyrexia model in rats. ${ }^{34)}$ Anti-inflammatory drugs that inhibit prostaglandin biosynthesis are capable of suppressing fever ${ }^{35)}$ or the inflammatory response seen after cerebral ischemia. ${ }^{36}$

In summary, our results in this study indicate that BE confers neuroprotection against transient ischemic brain injury through a mechanism that involves the reduction of NR1 expression. Although other mechanisms might also be involved, we believe that the reduction of calcium influxes via NR1 is main event accounting for neuroprotective properties of BE.

Acknowledgements The authors would like to thank Mr. Suek Han, Seung Uk Lee and Ms. Hyun Sook Kim for their technical help for this study. This work was supported by the grant from Biogreen 21 Program the rural development administration, Republic of Korea. The authors deeply appreciate this support.

\section{REFERENCES}

1) Kirino T., Brain Res., 239, 57-69 (1982).

2) Petito C. K., Pulsinelli W. A., J. Cereb. Blood Flow Metab., 4, 194 205 (1984).

3) Katoh A., Ishibashi C., Shiomi T., Takahara Y., Eigyo M., Brain Res., 577, 57-63 (1992).

4) Gustafson Y., Nilsson I., Mattsson M., Astrom M., Bucht G., Drugs Aging, 7, 298-309 (1995).
5) Ivanovska N., Philipov S., Int. J. Immunopharmacol., 18, 553-561 (1996).

6) Kupeli E., Koşar M., Yeşilada E., Başer C. J. C., Başer C., Life Sci., 72, 645-657 (2002).

7) Fukuda K., Hibiya Y., Mutoh M., Koshiji M., Akao S., Fujiwara H., J. Ethnopharmacol., 66, 227-233 (1999).

8) Shamsa F., Ahmadiani A., Khosrokhavar R., J. Ethnopharmacol., 64, 161-166 (1999).

9) Marin-Neto J. A., Maciel B. C., Secches A. L., Gallo Junior L., Clin. Cardiol., 11, 253-260 (1988).

10) Ricciopo N. F., Br. J. Pharmacol., 108, 534-537 (1993).

11) Wang Y. X., Zheng Y. M., J. Cardiovasc. Pharmacol., 30, 214-222 (1997).

12) Rothman S. M., Olney J. W., Ann. Neurol., 19, 105-111 (1986).

13) Choi D. W., Rothman S. M., Annu. Rev. Neurosci., 13, 171-182 (1990).

14) Takahara A., Konda T., Enomoto A., Kondo N., Biol. Pharm. Bull., 27, 1388-1391 (2004).

15) Kang T. C., Hwang I. K., Park S. K., An S. J., Yoon D. K., Moon S. M., Lee Y. B., Sohn H. S., Cho S. S., Won M. H., J. Neurocytol., 30, 945-955 (2001).

16) Won M. H., Kang T., Park S., Jeon G., Kim Y., Seo J. H., Choi E., Chung M., Cho S. S., Neurosci. Lett., 301, 139-142 (2001).

17) Hwang I. K., Eum W. S., Yoo K. Y., Cho J. H., Kim D. W., Choi S. H., Kang T. C., Kwon O. S., Kang J. H., Choi S. Y., Won M. H., Free Radic. Biol. Med., 39, 392-402 (2005).

18) Kirino T., Tamura A., Sano K. A., Stroke, 17, 455-459 (1986).

19) Dai R., Li K., Li Q., Bi K., J. Chromatogr. Sci., 42, 207-210 (2004).

20) Xuejiang W., Ichikawa H., Konishi T., Biol. Pharm. Bull., 24, 558563 (2001).

21) Ichikawa H., Konishi T., Biol. Pharm. Bull., 25, 898-903 (2002).

22) Yu S. J., Kim J. R., Lee C. K., Han J. E., Lee J. H., Kim H. S., Hong J. H., Kang S. G., Biol. Pharm. Bull., 28, 1016-1020 (2005).

23) Pettmann B., Henderson C. E., Neuron, 20, 633-647 (1998)

24) Nishizawa Y., Life Sci., 69, 369-381 (2001).

25) Legendre P., Rosenmund C., Westbrook G. L., J. Neurosci., 13, 674 684 (1993).

26) Okabe S., Miwa A., Okado H., J. Neurosci., 19, 7781-7792 (1999).

27) Ying Z., Babb T. L., Comair Y. G., Bingaman W., Bushey M., Touhalisky K., J. Neuropathol. Exp. Neurol., 57, 47-62 (1988).

28) McIlhinney R. A., Le Bourdelles B., Molnar E., Tricaud N., Streit P., Whiting P. J., Neuropharmacology, 37, 1355-1367 (1998).

29) Watanabe M., Fukaya M., Sakimura K., Manabe T., Mishina M., Inoue Y., Eur. J. Neurosci., 10, 478-487 (1998).

30) Huang C. G., Chu Z. L., Wei S. J., Jiang H., Jiao B. H., Thromb. Res., 106, 223-227 (2002).

31) Hui K. K., Yu J. L., Chan W. F., Tse E., Life Sci., 49, 315-324 (1991).

32) Hua Z., Wang X. L., Acta Pharm. Sin., 29, 576-580 (1994).

33) Wang F., Zhao G., Cheng L., Zhou H. Y., Fu L. Y., Yao W. X., Brain Res., 999, 91-97 (2004).

34) Yeșilada E., Kupeli E., J. Ethnopharmacol., 79, 237-249 (2002).

35) Flower R., Vane J. R., Nature (London), 240, 410 - 411 (1972).

36) Iadecola C., Alexander M., Curr. Opin. Neurol., 14, 89-94 (2001). 